\title{
SHORT-TERM ANTIEPILEPTIC TREATMENT OF SOLITARY CEREBRAL CYSTICERCOSIS
}

A prospective randomized study of short-term vs long-term antiepileptic drug (AED) treatment of 206 subjects with new onset seizures and solitary cerebral cysticercus granuloma (SCCG) was conducted at the Department of Neurology, Institute of Medical Sciences, Banaras Hindu University, Varanasi, India. Group A (98) patients were treated for 6 months and group B (108 patients) for 2 years. Patients were monitored during and 18 months or longer after tapering for 6 weeks and stopping treatment. Seizures were partial with or without secondary generalization in $80 \%$ of patients. Carbamazepine and phenytoin were used as first-line AEDs. Complete spontaneous resolution of the SCCG had occurred in $66.3 \%$ of group A patients and in $57.4 \%$ of group B, while the remaining patients showed punctated residual calcification on CT. Seizures recurred during the 18 month follow-up period in $3.1 \%$ and $4.8 \%$ of group A and B patients showing complete resolution of the granuloma (NS, $\mathrm{P}=0.61$ ). In those with calcified residua, seizures recurred in $42.4 \%$ and $21.7 \%$ of groups $\mathrm{A}$ and $\mathrm{B}$, respectively, and the difference was significant $(\mathrm{P}<0.05)$. Seizures responded when therapy was resumed in all patients with recurrences. (Verma A, Misra S. Outcome of short-term antiepileptic treatment in patients with solitary cerebral cysticercus granuloma. Acta Neurol Scand March 2006;113:174-177). (Respond: Dr Archana Verma, New L-23 Hyderabad Colony, Banaras Hindu University, Varanasi 221 005, India).

COMMENT. The study shows that solitary cerebral cysticercus granuloma with epilepsy is a self-limiting disease. In patients with complete resolution of the lesion on CT, early withdrawal of AED treatment after 6 months can be attempted without risk of seizure recurrence, whereas patients with persistent residual calcifications may require longer-term treatment.

Neurocysticercosis caused by infection of the CNS with Taenia solium is common in developing countries. It is rare in the USA but accounts for $25 \%$ of intracranial tumors in Mexico. A patient in India, a 16-year-old female, with a history of migraine, presented with headache that was prolonged, lasting longer than 72 hours (status migrainosus). Neurological examination was normal, with no papilledema. CT of the head showed hydrocephalus with disproportionate enlargement of the $4^{\text {th }}$ ventricle. MRI revealed a solitary cystic lesion with scolex in the $4^{\text {th }}$ ventricle that proved to be neurocysticercosis. Headache was partially relieved by IV mannitol and dexamethasone. Endoscopic removal of the cyst was unsuccessful because of adhesions. CT following $3^{\text {rd }}$ ventriculostomy revealed reduction in size of $4^{\text {th }}$ and lateral ventricles, and symptomatic relief. The patient was discharged on albendazole (400 mg BD orally for 14 days) and phenytoin $200 \mathrm{mg}$ PM. At 6-month followup, she had suffered no headaches or seizures; CT showed a calcified dot in the $4^{\text {th }}$ ventricle (Shukla R, Paliwal VK, Jha D. Headache Jan 2006;46:169-174).

In a long-term study of 240 patients with seizures and neurocysticercosis in Mexico, outcome was better in patients who received albendazole (Vasquez V, Sotelo J. N Engl J Med 1992;327:696-701). Seizures were fewer after medical treatment than after surgical excision of the cyst. (Ped Neur Briefs Oct 1992). 